\title{
UNIQUE HUES ARE NOT INVARIANT WITH BRIEF STIMULUS DURATIONS ${ }^{1}$
}

\author{
ALLEN L. NAGY ${ }^{2}$ \\ University of Michigan
}

(Receited 18 September 1978)

\begin{abstract}
Unique hue loci were obtained from three observers at several intensity levels with a 1 -sec stimulus flash and a $17 \mathrm{msec}$ stimulus flash. With the one-second stimulus the three spectral unique hues were approximately invariant but unique red was not. With the $17 \mathrm{msec}$ stimulus none of the unique hues is strictly invariant. These results are discussed in terms of their implications for the nature of the cone signal inputs to the opponent color mechanisms.
\end{abstract}

\section{INTRODUCTION}

The relationship between uniques hues and invariant hues has long been of interest in the study of color perception. Unique hues are the "psychologically" primary or "pure" hues, which do not appear to be compounds of other hues: red, green, yellow. and blue. The term invariant hues refers to those wavelengths or spectral composites whose perceived hue does not vary with changes in stimulus intensity. It is clear that the hue of most stimuli does vary with stimulus intensity (Purdy, 1931). The effect is commonly known as the Bezold-Brücke effect.

As early as 1880 Hering maintained that the set of unique hues was identical with the set of invariant hues. This identity has significance for models of the color coding mechanisms and explanations of the intensity dependence of most hues. The intensity dependence of hue has been considered to be a manifestation of non-linearity in the color coding mechanism nearly since the time of its discovery. This idea is generally credited to Pierce (1887), though some credit should be given to Maxwell (1856) for first recognizing the non-linearity of color coding. However, there has been some debate about where the non-linearity occurs. Trichromatic theories of color coding have suggested that the hue shifts occur as a result of non-linearities in the signals from the three cone mechanisms (Pierce, 1877; Walraven, 1961; Savoie, 1973). Opponent theories of color coding have suggested that the signals input to the opponent mechanisms from the three cone mechanisms are linear with intensity, and that the non-linearity which produces the hue shifts occurs in the signals of the opponent mechanisms (Judd, 1951; Jameson and Hurvich, 1955). Some resolution to this debate has

1 This research was supported by NEI Post Doctoral Fellowship 7 F32 EY.05083 to the author, and NSF grant 33642-X to David H. Krantz. Portions of this paper were presented at the Spring Meetings of the Association for Research in Vision and Opthalmology. Sarasota. Florida 1978.

2 Current address: Center for Human Information Processing, University of California. San Diego. California 92093 , U.S.A. recently been provided by Krantz (1975) and Larimer et al. (1974, 1975), whose work revolves around the question of whether unique hues are invariant hues and whether additions of hues unique with respect to the same mechanism (e.g. unique yellow and unique blue, which both are unique with respect to the red/ green mechanism) result in another unique hue. These two properties follow from the assumptions of modern opponent colors theory (Jameson and Hurvich, 1955; Krantz, 1975), which include:

(i) Linear recombination of signals initiated by photopigment absorptions in cones into two opponent color mechanisms; red/green and yellow/blue.

(ii) Faster growth of yellow/blue opponent mechanism signals than red/green signals as intensity is increased.

(iii) Dependence of perceived hue on the ratio of the two opponent mechanisms responses and in particular, observance of unique hues when the response of one opponent mechanism is zero.

Together these assumptions predict that all unique hues must be invariant hues and conversely that all invariant hues must be unique hues. Additions of two hues unique with respect to the same mechanism must produce another unique hue. All hues which are not unique must vary with stimulus intensity as a result of the faster growth of yellow/blue opponent mechanism signals.

From their experiments Larimer et al. conclude that assumption (i) is correct for the red/green mechanism but not for the yellow/blue mechanism. They found that unique red was not invariant, which implies that signals input to the yellow/blue mechanism are not linear functions of intensity. They point out. however, that the departure from linearity would tend to work against the Bezold-Brücke hue shifts. This finding implies that the Bezold-Bruicke hue shift is primarily a manifestation of non-linearity more proximal than the site of opponent cancellation, as suggested by assumption to (ii). Though there is some non-linearity in input to the yellow/blue mechanisms, its effect on perceived hue must be small compared to the effect of the non-linearity at the opponent level which primarily determines how the hue of most stimuli will change with intensity. 
Recent experiments (Savoie. 1973; Nagy and Zacks. 1977: Genter et al. 1978) have suggested that unique yellow is not invariant with intensity when the stimulus is a brief flash. This result implies that assumption (i) may not hold for the red green mechanism as well as the yellow blue mechanism under some conditions. It also suggests that the degree of linearity or nonlinearity of cone signals input to the opponent mechanisms may be dependent to some degree on the particular stimulus conditions used. This paper presents further evidence for this suggestion. Results show that the invariance of unique hues is clearly dependent on stimulus duration. implying that the nature of the intensity-response functions of the signals input to the opponent mechanisms is also dependent on stimulus duration.

\section{METHODS}

A conventional Maxwellian view optical system was used to deliver stimuli to the observer. A Xenon-arc lamp (150 W Osram) driven by a current-regulated d.c. power supply provided the illumination which was passed through Schoeffel double monochromators. Temporal parameters were controlled with electronic shutters (Vincent Uniblitz) which provided nearly square-wave pulses either $17 \mathrm{msec}$ or $1 \mathrm{sec}$ in duration. Intensity was controlled with calibrated Wratten neutral density wedges. Radiance measures were made with a photodiode (United Detector Technology Pin-10) and illuminance at $580 \mathrm{~nm}$ was estimated with a MacBeth Illuminometer and the method of Westheimer (1966). A field stop provided a stimulus approx 0.6 in diameter. An adjustable bite bar was used to hold the observer rigidly in position and fixation was controlled with four small, dim fixation points arranged in a diamond shape. The stimulus was presented at the intersection of imaginary lines connecting the horizontal and vertical pairs. The observer was instructed to fixate the imaginary intersection point in order to ensure that the stimulus was presented to the rod free fovea. Two considerations were involved in the choice of the field size. A small field was desirable to ensure that the stimulus was presented to a rod free area. in order to avoid possible effects of rod signals on hue at low illuminance levels. Second, it seemed desirable to use a field size small enough to allow the simultaneous presentation of two fields to the rod free area, so that data obtained with a hue matching method could be compared to results obtained with the method described here. (Hue matching data will be reported in a later paper.) The $36^{\circ}$ field was chosen since it was small enough to meet these considerations, yet large enough to produce trichromatic color perception with the procedures used here. Small field tritanopia is generally found with somewhat smaller fields and appears to occur only with strict continuous fixation of a long duration stimulus. Bedford and Wyszecki (1958) have shown that wavelength discrimination in the blue region of the spectrum is nearly as good with a 12 field as it is with a 1 field if the observer is allowed to scan the visual field. and Ingling et al. (1970) have shown that observers are trichromatic even with 3 fields presented to the central fovea if the stimulus is a brief flash

Experiments were run in a darkened room with the observer dark-adapted prior to the experiment. On each trial the observer wals forced to make a binary decision about the hue of the test stimulus. For example. if the objective was to determine unique yellow or blue. the observer was asked to respond red or green on each trial to indicate whether the stimulus contained some redness or greeness. Similarly, for unique red or green. the observer was required to indicate whether the stimulus contained blueness or vellowness. Wavelength was varied according to the rules of a double random starcase procedure (Cornsweet. 1962). The starling points of the two randomly intermixed staircases, generally 30 or $\$ 0 \mathrm{~nm}$ apart. were chosen to bracket the region containing the unique hue Each staircase independently followed the same rules for step sizes. The initial step size was $10 \mathrm{~nm}$. After the first reversal, the step size was $5 \mathrm{~nm}$ until the second reversal occurred. After the second reversal, the step size was fixed at either two or three $\mathrm{nm}$. The staricase was terminated when three reversals had occurred with the smallest step size. After both staircases were completed the luminance of the stimulus was changed and another pair of staircases was run. Luminance was varied in $0.55 \mathrm{log}$ unit steps over the available range generally going from bright to dim or dim to bright. Trials were presented every $10-20$ sec with brief rest periods between pairs of staircases. In order to obtain a unique red. 625 and $470 \mathrm{~nm}$ lights were mixed. The $625 \mathrm{~nm}$ light was yellow-red in hue and the $470 \mathrm{~nm}$ light was nearly unique blue for most observers. Addition of small amounts of $470 \mathrm{~nm}$ light to the $625 \mathrm{~nm}$ light cancelled out the rellowness in the $625 \mathrm{~nm}$ light resulting in a unique red. which was quite saturated in chromatic appearance. Slightly more saturated reds could have been produced by adding the $470 \mathrm{~nm}$ light to an even longer wavelength yellow-red. but even smaller amounts of the $470 \mathrm{~nm}$ light would have been required to cancel the yellowness, making the task difficult for the observer at low luminance levels. Therefore $625 \mathrm{~nm}$ was chosen as one primary since its mixture with $470 \mathrm{~nm}$ resulted in a unique red with good chromatic saturation and measurements could be obtained at relatively low luminance levels with little difficulty. The luminance of the $625 \mathrm{~nm}$ light was fixed at a given level and the luminance of the $470 \mathrm{~nm}$ light was varied with the staircase procedure described above. The starting points of the two staircases were $1.8 \mathrm{log}$ units apart and successive step sizes in the staircase were 0.4 . 0.2. and 0.1 log units.

One female. and two males. including author AN. served as observers. All had normal color vision and extensive experience with the judgment before data presented here were collected.

\section{RESLLTS}

Determination of unique hues with $I$ sec flashes are shown in Fig. 1 and those with $17 \mathrm{msec}$ flashes are shown in Fig. 2. Each plotted point is the mean of at least three daily means which were obtained by averaging the last three reversal points in each member of a pair of staircases for a given condition. The horizontal lines indicate $80^{\circ}$ confidence intervals based on between-day variability. Note that the abscissa for unique blue, green, and yellow is wavelength, but the abscissa for unique red is the ratio of the illuminances of the 625 and $470 \mathrm{~nm}$ lights required to produce unique red. The unique red data were plotted in this way to make them more easily comparable to the other data. A larger ratio indicates that less blue light is needed to cancel yellowness in the $625 \mathrm{~nm}$ light and a smaller ratio indicates that more blue is needed. This parallels changes on the wavelength abscissa where an increase in the wavelength for unique green implies that less blue is needed to cancel the yellowness and a decrease in wavelength implies that more blue is needed to cancel the yellow.

Consider the results obtained with a 1 sec flash in Fig. 1. Unique blue and unique yellow appear to be invariant with intensity for all three observers Unique red is clearly not invariant. The 625/470 illuminance ratio must be increased with increasing in - 


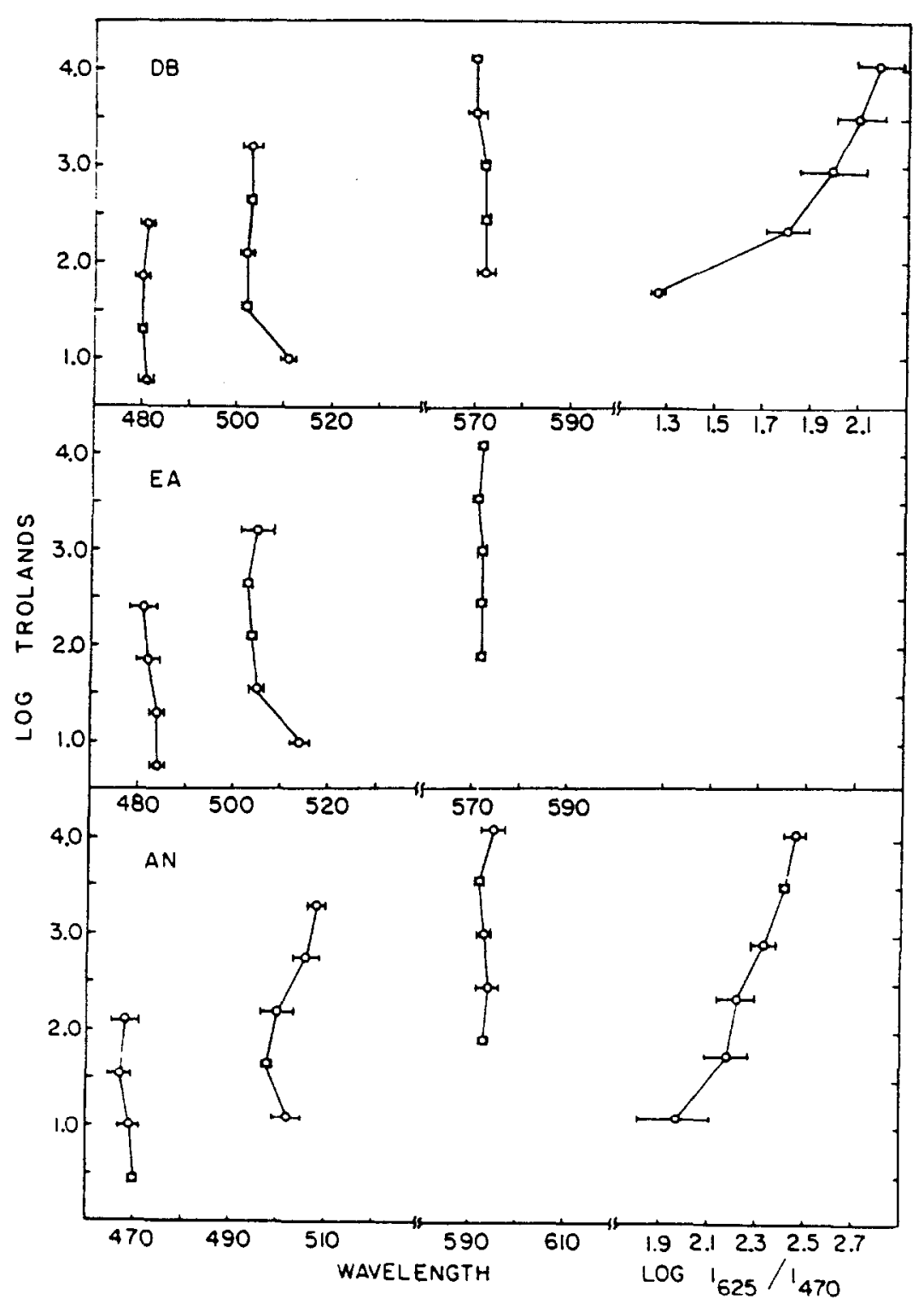

Fig. 1. Unigue hue loci with a 1-sec stimulus flash.

tensity to maintain unique red for each observer. That is, a smaller proportion of blue light is needed to cancel the yellowness in the $625 \mathrm{~nm}$ light as intensity is increased. Unique green also appears to vary with intensity for each observer though the trend is smaller and not consistent across observers. For DB and EA wavelength must be increased to obtain unique green at the lowest intensity. For AN wavelength must be increased as intensity is increased.

Next. consider the results obtained with the $17 \mathrm{msec}$ flash in Fig. 2. All four unique hues shift or lack invariance with intensity. The only case where there is not a significant shift is unique blue for observer $\mathrm{DB}$, but the trend in $\mathrm{DB}^{\circ} \mathrm{s}$ unique blue data is similar to that of the other observers. The shifts in unique blue and yellow appear to be small in comparison with the shifts of unique red and green. Also. note that the shifts for unique red and green are in opposite directions. As intensity is increased. wavelength must be decreased to maintain unique green, indicating that more blue is needed to cancel out yellowness. At the highest intensity there is a suggestion of a reversal of this trend. To maintain unique red as intensity is increased, the proportion of blue light must be decreased in order to just cancel yellowness in the $625 \mathrm{~nm}$ light.

The dashed lines in Fig. 2 indicate the approximate spectral locations of the unique hues with $1 \mathrm{sec}$ flashes from Fig. 1. It appears that use of the shorter duration has not dramatically shifted the locations of the unique hues, implying that the relative weighting of the input signals from cone mechanisms to the opponent mechanisms has not been much affected by stimulus duration.

\section{DISCLSSION}

The results described above show that the invariance or lack of invariance of unique hues is dependent on stimulus duration. With $1-\sec$ flashes. unique 


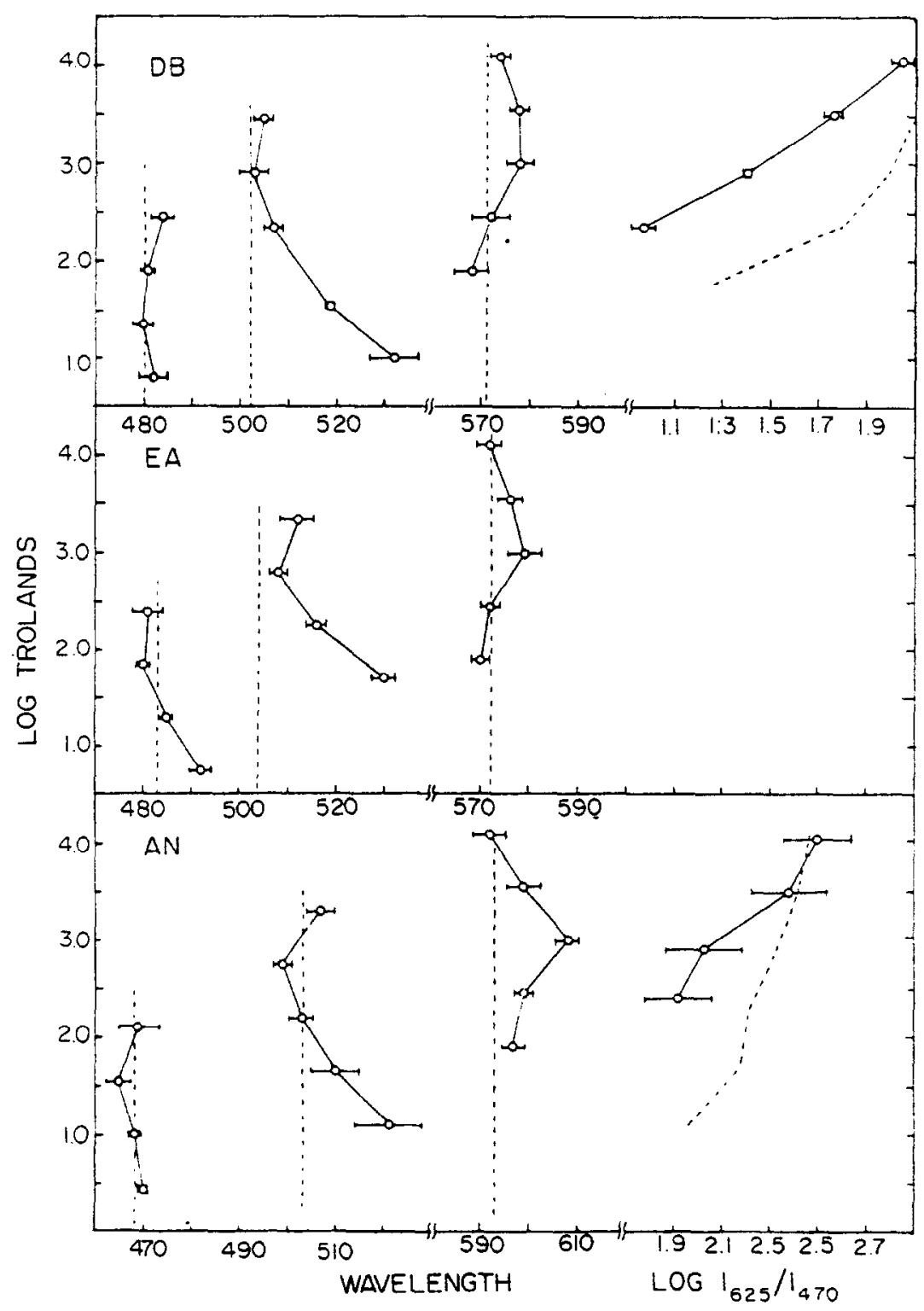

Fig. 2. Solid lines indicate unique hue loci with a $17 \mathrm{msec}$ stimulus flash. Dashed lines indicate unique hue loci with a $1-\mathrm{sec}$ flash from Fig. 1.

blue and unique yellow are quite invariant with changes in intensity. Unique green is approximately invariant but unique red is clearly not invariant. These results agree with previous experiments by Larimer et al. (1974. 1975) with $1-\mathrm{sec}$ flashes and are consistent with their conclusion that cone signals input to the red/green opponent mechanism are linear with intensity while those input to the yellow/blue mechanism are not. Purdy's early experiments (1931) with uncontrolled stimulus duration also produced similar results suggesting that this conclusion may hold for longer stimulus durations as well. However, when a $17 \mathrm{msec}$ stimulus duration is used, all four unique hues show consistent departures from invariance, implying that cone signals input to both opponent mechanisms are non-linear functions of intensity. The transition from short flash behavior to long-flash behavior has not been determined in these experiments, but results reported by Nagy and Zacks (1977) sug- gest that the transition occurs at durations shorter than 300 msec.

It might be argued that temporal summation should be taken into account in comparing results obtained with the $17 \mathrm{msec}$ flash to those obtained with the 1 -sec flash. It is clear that the visual system integrates energy over some period of time, and thus the relevant comparison should be made on a scale of energy per unit of integration time, if the integration time is longer than $17 \mathrm{msec}$. For example, Barlow's experiments on increment thresholds for rods suggest that the visual system integrates energy over approximately $100 \mathrm{msec}$ at thresholds levels (see Barlow. 1958). If this integration time applied to suprathreshold stimuli as well, the the $1-\mathrm{sec}$ stimulus would be more effective than the $17 \mathrm{msec}$ stimulus by a factor of $100 / 17$ or $0.76 \log$ units. The data obtained with the $17 \mathrm{msec}$ flashes should then be slid downward on the log Troland scale by $0.76 \log$ units for comparison 
with the data obtained with the 1-sec flash. However. there is clear evidence that the integration time for suprathreshold stimuli is much shorter than the integration time at threshold energy levels. Aiba and Stevens (1964) have found that the interval of complete temporal summation of energy for the brightness of suprathreshold flashes is approximately $10 \mathrm{msec}$ for a dark-adapted observer at photopic levels. If integration time is taken to be only $10 \mathrm{msec}$, data obtained with $17 \mathrm{msec}$, and $1-\mathrm{sec}$ flashes are directly comparable without shifting on the log Troland scale since both stimuli are longer than the integration interval. Still, it might be argued that the results obtained by Aiba and Stevens apply only to the brightness or luminance mechanism, and that temporal summation in the color coding mechanism should clearly occur over a longer interval than in the brightness mechanism (see for example, Regan and Tyler, 1971). The relevant integration interval for the results presented above would appear to be the interval of complete summation for suprathreshold signals input to the opponent mechanisms. At present, no estimate of this interval appears to exist in the literature, so no attempt has been made to shift the curves on the basis of an integration argument. In view of the fact that the integration time for suprathreshold signals appears to be fairly short, at least for suprathreshold brightness signals, it seems unlikely that taking integration time into account could explain the difference between results obtained with short and long flashes by shifting them to non-overlapping ranges. Even if the short flash data are shifted downward by $0.76 \log$ units, which corresponds to an integration time of $100 \mathrm{msec}$, there is still a substantial range of overlap between the short flask curves and the $1 \mathrm{sec}$ curves and the results are clearly different at comparable levels. Therefore, other possible explanations of the short flash non-linearity are discussed below.

\section{Possible sources of non-linearity}

It is perhaps not surprising that the function relating response to intensity is dependent upon stimulus duration, for such a dependence has been shown in both physiological recordings from vertebrate receptors (Fain and Dowling, 1973; Baylor et al. 1974; Boynton and Whitten, 1970; Normann and Werblin. 1974) and in human psychophysical experiments on brightness (Mansfield, 1973). What is surprising is that the nature of this dependence seems to be very different for color signals on the one hand, and receptor and brightness signals on the other. Unique hue experiments suggest that signals input to the opponent mechanisms are not linear with short flashes, but more nearly linear with long flashes, at least for the $\mathrm{red} / \mathrm{green}$ mechanism. The experiments on receptors and brightness cited above suggest that signals are nearly linear with short flashes and non-linear with long flashes.

If conclusions drawn from the vertebrate receptor recordings apply to human receptors, we are left with the problem of explaining the source of the nonlinearity implied by the short flash unique hue data and also how the receptor non-linearity is cancelled out for the red/green mechanism in the long flash case.
Two possible sources of non-linearity in the short flash case are the neural links between the receptor and the site of opponent cancellation, and the interaction of the cone signals at the site of cancellation. The signals generated in cones presumably have to pass through some anatomical elements before they reach the site of opponent cancellations. Thus the nonlinearity may occur in a pathway between the receptor and this site.

The second possibility suggests that the signals reaching the site of cancellation are linear with intensity but they interact non-linearly. possibly as a result of the briefness of the signal and differences in arrival time at the site of cancellation. There is evidence that the latency of response of a cone mechanism is related to its strength of excitation (Alpern, 1954: Drum, 1977) and also that the latency may be different for different mechanisms (Green, 1969; deLange, 1958; Kelly, 1974; Walraven and Leebeek, 1964; Cicerone, 1974). Thus, it is possible that brief signals from the individual cone mechanisms arrive at the opponent cancellation site at slightly different times and are summed or differenced non-linearly as a result. However, at present neither of these explanations seems capable of reconciling long flash additivity and invariance of unique hues with non-linearities found in physiological recordings from vertebrate receptors with long flashes.

\section{Inputs to red/green and yellow/blue mechanisms}

Some inferences can be drawn about the nature of the input signals to the opponent mechanisms from the short flash unique hue data. Over the lower range of intensitites the shifts of unique yellow and unique blue both indicate that the input to greeness from the middle wavelength cones grows at a slightly greater rate than the input to redness from the short and long wavelength cones as intensity in increased. At the highest intensities of unique yellow the relationship appears to reverse and the input to redness grows faster than the input to greeness. The shifts of unique green and unique red are in opposite directions. That is, the shift of unique green suggests that the input to yellowness grows at a greater rate than the input to blueness while the shift of unique red suggests the opposite relationship. This result implies that the input to yellowness from the middle wavelength cones grows at a greater rate than the input to blueness from the short wavelength cones, which in turn grows at a greater rate than the input to yellowness from the long wavelength cones.

\section{SUMMARY}

The results imply that the properties of signals input to the opponent mechanisms are to some degree dependent on stimulus conditions. This finding imposes some limitation on the generality of opponent mechanism properties measured under the more typical experimental conditions of color vision experiments. Hopefully these limitations are not severe and occur only under extreme conditions such as the very brief flashes used here. Until these limits are further explored some care should be taken in generalizing properties determined under one set of conditions to another set of conditions. 
The dependency of the intensity-response function on stimulus duration implied by the unique hue data is consistent with evidence from physiological recordings from receptors and psychophysical experiments on brightness. However, the nature of the relationship between the response function and stimulus duration implied by the unique hue data appears to be nearly opposite that found in other types of experiments. This raises some perplexing problems that remain to be resolved.

Acknowledyements-I would like to thank $\mathrm{Dr}$ David Krantz. whose advice and support were invaluable in all phases of this work: and Dr R. M. Boynton for his comments on a earlier version of this manuscript.

\section{REFERENCES}

Aiba T. S. and Stevens S. S. (1964) Relations of brightness to duration and luminance under light-and dark-adaptation. Vision Res. 4, 391-401.

Alpern M. (1954) The relation of visual latency to intensity A.M.A. Arch. Opth. 51, 369-374.

Barlow H. B. (1958) Temporal and spatial summation in human vision at different background intensities. $J$. Physiol. Lond. 141, 337-350.

Baylor D. A.. Hodgkin A. L. and Lamb T. (1974) The electrical response of turtle cones to flashes and steps of light. J. Physiol. Lond. 242, 759-791.

Bedford R. E. and Wyzsecki G. W. (1958) Wavelength discrimination for point sources. J. opt. Soc. Am. 48. 129-135.

Boynton R. M and Whitten D. N. (1970) Visual adaptation in monkey cones: Recordings of late receptor potentials. Science, N.Y. 1423-1426.

Cicerone C. M. O. (1974) Theory of luminance detection and measurement of the impulse responses and frequency responses of the color mechanisms of the eye. Mich math. Psychol. tech. Rep. 74-6.

Cornsweet T. N. (1962) The staircase method in psychophysics. Am. J. Psychol. 75, 485-49!.

Drum B. A. (1977) Cone interactions at high flicker frequencies: Evidence for cone latency differences? $J$. opt Soc. Am. 67, 1601-1603.

Fain G. L. and Dowling J. E. (1973) Intracellular recordings from single rods and cones in the mudpuppy retina. Science, N.Y. 180. 1178-1181.

Genter C. R., Geri G. A. and Kandel G. L. (1978) Luminance dependent shifts in unique yellow. Abstract in Supplement to Incest. Opthal. and Visual Science, April, 1978.
Green D. G. (1969) Sinusoidal flicker characteristics of the color-sensitive mechanisms of the eye. Vision Res. 9. $591-601$.

Ingling C. R. Scheibner H. M. O. and Boynton R. M. (1970) Color naming of small foveal fields. Vision Res. $10,501-511$.

Jameson D. and Hurvich L. (1955) Some quantitative aspects of an opponent colors theory-II. Brightness. saturation, and hue in normal and dichromatic vision. $J$. opt. Soc. Am. 45, 602-616.

Kelly D. H. (1974) Spatio-temporal frequency characteristics of color vision mechanisms. J. opt. Soc. $4 \mathrm{~m}$. 64, $983-990$.

Krantz D. H. (1975) Color measurement and color theory -II. Opponent colors theory. J. math. Psychol. 12. $304-337$.

Lange $H$. de (1958) Research into the dynamic nature of the human fovea-shift in brightness and delay in color perception. J. opt. Soc. Am. 48, 784-789.

Larimer J.. Krantz D. H. and Cicerone C. M. (1974) Opponent-process additivity: red/green equilibria. Vision Res. 14. $1127-1140$.

Larimer J.. Krantz D. H. and Cicerone C. M. (1975) Opponent-process additivity-II. Yellow/blue equilibria and non-linear models. Vision Res. 15, 723-732

Mansfield R. J. W. (1973) Brightness function: effect of area and duration. J. Opt. Soc. Am. 63, 913-920.

Maxwell J. C. (1856) Experiments on color as perceived by the eye with remarks on colour blindness. Trans. R. Soc. Edinb. reprinted in The Scientific Papers of James Clerk Maxwell (Edited by Niven W. D.). Dover, New York.

Nagy A. L. and Zacks J. L. (1977) The effects of psychophysical procedure and stimulus duration in the measurement of Bezold-Brücke hue shifts. Vision Res. 17. 193-200

Normann R. A. and Werblin F. S. (1974) Control of retinal sensitivity-I. Light and dark adaptation of vertebrate rods and cones. J. gen. Physial. 63, 37-61.

Pierce C. S. (1877) Note on the sensation of color. Am. J. Sci. Ser. 3, 13, 247.

Purdy D. M. (1931) Spectral hue as a function of intensity. Am. J. Psychol. 43, 541-559.

Regan D. and Tyler C. W. (1971) Temporal summation and its limit for wavelength changes: an analog of Bloch ` Law for color vision. J. opt. Soc. Am. 61, 1414 1421.

Savoie R. S. (1973) Bezold-Brücke effect and visual nonlinearity. J opt. Soc. Am. 63, 1253-1261.

Walraven P. L. (1961) On the Bezold-Brücke phenomenon J. opt. Soc. Am. 51, 1113-1116.

Walraven P. L. and Leebeek H. J. (1964) Phase shift of sinusoidally alternating colored stimuli. $J$. opt. Soc. Am. 54, 78-82.

Westheimer G. (1966) The Maxwellian view. Vision Res. 6, 669-68?. 